\title{
CONCEPCIONES DE LA GEOMETRÍA ANALÍTICA EN LOS LIBROS DE TEXTO DE SECUNDARIA Y UNIVERSIDAD EN ESPAÑA DURANTE EL SIGLO XIX
}

\section{Conceptions about the Analytic Geometry in the secondary and university Spanish textbooks during the nineteenth century}

\author{
Isabel M. ${ }^{a}$ SÁnchez Sierra \\ IES Francisco Salinas. Salamanca \\ Correo-e: isamss@yahoo.com \\ M. ${ }^{a}$ Teresa GonzÁlez Astudillo \\ Departamento de Didáctica de la Matemática y Didáctica de las Ciencias Experimentales \\ Universidad de Salamanca \\ Correo-e:maite@usal.es
}

Recepción: 28 de septiembre de 2020. Envío a informantes: 30 de septiembre de 2020 Aceptación definitiva: 3 de noviembre de 2020

Resumen: Este artículo muestra las concepciones acerca de la Geometría Analítica que aparecen en los libros de texto utilizados en España durante el siglo XIX y que se extraen de la presentación de los conceptos matemáticos en dichos libros. Mediante el análisis de contenido de diez obras hemos identificado cuatro términos para referirse a ella: Aplicación del Álgebra a la Geometría, Geometría Analítica, Análisis determinada y Análisis indeterminada. Y dos concepciones: la primera de ellas es considerarla como un método de resolución de problemas geométricos por medio del Álgebra, de manera similar a como se utiliza para resolver problemas aritméticos, pero operando con segmentos en vez de operar con cantidades y la segunda, como un método de resolución de problemas geométricos por medio del Álgebra utilizando sistemas de coordenadas.

Palabras clave: Geometría Analítica; Concepciones; Historia de la educación; libros de texto. 
Aвstract: This article shows the conceptions about the Analytical Geometry held in the textbooks used in Spain during the XIX century. These conceptions are extracted from the presentation of the mathematics concepts in these books. Through the content analysis of ten works we have identified four terms to refer to it (Application of Algebra to Geometry, Analytical Geometry, Determinate Analysis and Indeterminate Analysis. And two conceptions: the first one is to consider it as a method for solving geometric problems by means of Algebra, like the method used to solve arithmetic problems, but instead of operating with quantities operating with segments, the second one as a method of solving geometric problems by means of Algebra, using coordinate systems.

KeYwords: Analytic Geometry; Conceptions; History of education; Textbooks

\section{Introducción}

Y

A A FinAles Del siglo XViII encontramos libros didácticos en España que incluyen la Geometría Analítica como un contenido. Tal es el caso del libro de Benito Bails titulado Elementos de Matemáticas (I775-I78I). Pero es esencialmente durante el siglo XIX cuando se generaliza la enseñanza de esta rama de las Matemáticas y, por tanto, se puede analizar la evolución que sufrió hasta su consideración tal como la entendemos actualmente.

Pero ¿qué entendemos por Geometría Analítica? Según González (2007: 205):

La Geometría Analítica es un poderoso instrumento de ataque de los problemas geométricos que utiliza como herramienta básica el Álgebra. La esencia de su aplicación en el plano es el establecimiento de una correspondencia entre los puntos del plano y pares ordenados de números reales, es decir, un sistema de coordenadas, lo que posibilita una asociación entre curvas del plano y ecuaciones en dos variables, de modo que cada curva del plano tiene asociada una ecuación $f(x, y)=0$ y, recíprocamente, para cada ecuación en dos variables está definida una curva que determina un conjunto de puntos del plano, siempre respecto a un sistema de coordenadas.

Por su parte, Gaita (20I4: 29) considera la Geometría Analítica como:

El campo de estudio de la matemática que emplea un sistema de referencia y ecuaciones algebraicas y en donde se dice que un problema ha sido resuelto cuando se obtienen ecuaciones de curvas asociadas a dicho sistema de referencia.

Es decir, en la actualidad no concebimos esta rama de la Geometría sin el uso de sistemas de coordenadas, que nos permiten asociar a una curva una ecuación algebraica. Pero, como veremos, esta concepción dista mucho de sus orígenes, siendo la evolución larga y manteniéndose algunas reminiscencias de sus inicios en pleno siglo xix. En este trabajo pretendemos identificar las distintas concepciones 
de Geometría Analítica que se pueden encontrar en los libros de texto españoles del siglo XIX.

Este estudio se enmarca en la línea de investigación de la historia de las disciplinas escolares. Viñao (2006), comentando los inicios de las investigaciones realizadas por Goodson (I995) en esta línea de investigación, considera «las disciplinas escolares como algo no dado sino construido, como un producto social e histórico» (p. 248) y Goodson (1995) señala que esta línea debe «arrojar luz sobre la realidad contemporánea» (p. 27) en el sentido de que estos estudios históricos nos ayudan a comprender el presente para actuar en el futuro. Por tanto, las disciplinas escolares son fruto de una selección realizada históricamente y la comprensión de su evolución requiere un análisis histórico del proceso por el cual fueron construidas. Un elemento clave, aunque no el único, para determinar dicha evolución es el análisis de los libros de texto.

Un aspecto a tener en cuenta es la noción de evolución asociada al surgimiento de las disciplinas escolares en el sentido de que no son algo estático, sino que van cambiando por presiones sociales, influencias entre países o modificaciones en el conocimiento científico. Incluso, la propia práctica escolar hace que los contenidos se adapten a la realidad de las aulas y a las necesidades del momento. De hecho, Viñao (2006) plantea que se puede hablar de «asignatura, materia, actividad, ejercicio» (p. 263) antes de que se configure en disciplina, sugiriendo en ese caso utilizar el término de protodisciplina. Analizando los planes de estudio se puede comprobar efectivamente cómo la Geometría Analítica pasó de formar parte de los programas y contenidos de otras materias a llegar a tener una entidad propia (Sánchez, 2015).

Para comprender esta evolución hay que tener en cuenta que cada disciplina posee un código disciplinar que establece las reglas, pautas y formas de considerar el conocimiento en su seno, que se transmite de generación en generación y que está configurado por unos contenidos, el discurso sobre su utilidad y el de las prácticas docentes y académicas (Viñao, 2006). Estos códigos suelen hacerse visibles de muchas formas, pero en los libros de texto se pueden identificar claramente en los prólogos, los temas, los ejercicios o la secuenciación de los contenidos.

Por todo ello consideramos pertinente una investigación histórica sobre la presencia de la Geometría Analítica en los libros de texto de nuestro país, en el siglo XIX, que nos permita comprender y explicar la evolución sufrida a lo largo de este siglo a partir de las concepciones que muestran los autores de los libros de texto y que se manifiestan a través de un código disciplinar.

Comenzaremos la exposición haciendo un breve estudio de los orígenes de la Geometría Analítica para situar el problema de investigación y poner de manifiesto la forma de hacer de los matemáticos en los orígenes de esta rama de las matemáticas. A continuación, justificaremos la importancia del libro de texto como fuente documental en este tipo de investigaciones y explicaremos la metodología seguida en el análisis de los textos para centrarnos posteriormente en el estudio de las concepciones de la Geometría Analítica. 
CONCEPCIONES DE LA GEOMETRÍA ANALÍTICA EN LOS LIBROS DE TEXTO DE SECUNDARIA Y UNIVERSIDAD EN ESPAÑA DURANTE EL SIGLO XIX ISABEL M. ${ }^{a}$ SÁNCHEZ SIERRA Y M. ${ }^{a}$ TERESA GONZÁLEZ ASTUDILLO

\section{Los orígenes de la Geometría Analítica}

Podemos encontrar algunos vestigios del uso de sistemas de referencia o representaciones similares a las coordenadas actuales en la obra de Apolonio de Perga (262-190 a. C.?) (González, 2007) y en la de Nicolás de Oresme (c. 1323I382) (Chica, 200I); así como el uso del álgebra para la resolución de problemas geométricos por matemáticos árabes e indios en el siglo IX, como puede verse en la obra de Al-Khwarizmi (Chica, 20oI), pero no de forma general y sistemática. A diferencia de los matemáticos anteriores, tanto Descartes como Fermat desarrollaron un método general para la resolución de problemas geométricos mediante la aplicación del Álgebra. En un principio será Descartes quien pase a la historia como el padre de la Geometría Analítica gracias a su Geometría (1637), a pesar de que la geometría que describe en esta obra se parece poco a lo que hoy consideramos como Geometría Analítica. Más similar a la concepción actual es la que Fermat desarrolla en su libro Ad locos planos et solidos isagoge (1679), obra que fue publicada póstumamente (Fermat, I89I-19I2), aunque fue escrita antes de la aparición de La Geometría, por lo que en la actualidad se le reconoce igual mérito que a Descartes.

La geometría de Descartes se asemeja, en ciertos aspectos, a la Geometría Analítica actual, ya que esta deriva de aquella, pero también existen importantes diferencias entre las dos. La más importante es la interpretación que hace Descartes de las letras y los números que forman las ecuaciones; mientras que actualmente se considera que tanto los parámetros como las incógnitas representan cantidades, Descartes considera que representan segmentos de rectas. Esto conlleva una serie de problemas: por una parte, es necesario que las fórmulas sean homogéneas -principal problema que tuvieron los geómetras antiguos para aplicar el Álgebra a la Geometría- ya que, si a representa un segmento, $a^{2}$ representa un área y $a^{3}$ un volumen, por tanto, la expresión $a^{2} b^{2}-b$ no tiene sentido. Descartes solucionará este problema introduciendo el concepto de segmento unidad, que utiliza de forma implícita, así, en la expresión $\alpha^{2} b^{2}-b$ debemos considerar la cantidad $a^{2} b^{2}$ dividida una vez por la unidad, y la cantidad $b$ multiplicada dos veces por la unidad» (Descartes, 1637, trad. 1947: 52) lo que le permite operar libremente con expresiones de distinta dimensión.

Por otra parte, esta interpretación de las ecuaciones le lleva a ignorar las soluciones negativas por considerarlas falsas; hemos de tener en cuenta que, bajo su planteamiento, lo que se obtendrían serían segmentos de longitud negativa.

Otra importante diferencia entre el modo de hacer de Descartes y el actual se encuentra a la hora de dar la solución de un problema. Actualmente, una vez que se ha obtenido la solución algebraica simplemente se da su interpretación geométrica, sin embargo, Descartes construye, a partir de la solución algebraica, la solución geométrica con regla y compás como se venía haciendo hasta entonces.

Como veremos en este artículo muchas de estas características seguirán presentes en la Geometría Analítica que se estudiaba en España en el siglo XIX. 
CONCEPCIONES DE LA GEOMETRÍA ANALÍTICA EN LOS LIBROS DE TEXTO DE SECUNDARIA Y UNIVERSIDAD EN ESPAÑA DURANTE EL SIGLO XIX

ISABEL M. ${ }^{a}$ SÁNCHEZ SIERRA Y M. ${ }^{a}$ TERESA GONZÁLEZ ASTUDILLO

\section{Metodología de investigación}

El libro de texto fue durante años un objeto de estudio olvidado por los historiadores en educación, sin embargo, son múltiples los trabajos que han puesto de manifiesto la importancia del manual escolar (Delgado, I983; Choppin, 2000; Puelles, 2000; Collados, 2008). Esta línea se fundamenta en que la realidad educativa está guiada más por los libros de texto que por las tendencias curriculares o los planes de estudios (Schubring, 1987). Desde el punto de vista de la historia de la educación el libro de texto es una fuente inestimable ya que permite conocer las opiniones e ideas de sus autores o editores, encontrar sus orientaciones metodológicas, sus concepciones pedagógicas y los autores a los que se remiten (Delgado, 1983). Por otra parte, el manual escolar indica cómo ha sido llevada a la práctica la política educativa de un país, ya que selecciona, prioriza e impone unos contenidos frente a otros, así como una determinada forma de transmitirlos (Collados, 2008).

Este trabajo se ha realizado siguiendo la metodología de investigación histórica que incluye las siguientes fases: heurística, crítica, hermenéutica y síntesis (Ruiz, 1997). La heurística o búsqueda de fuentes documentales se centró en la búsqueda y selección de libros de texto del siglo xix en los que aparece la Geometría Analítica.

Para llevar a cabo esta selección se tuvo en cuenta el contexto histórico y educativo del país. En I836 surge, al menos sobre el papel, la segunda enseñanza, aunque en la práctica el número de institutos es muy escaso. En esta etapa no se publican listas oficiales de libros de texto, pero existen un gran número de manuales con el título Elementos de matemáticas, que es como se denominan las asignaturas de Matemáticas en los planes de estudios de segunda enseñanza (Vea, 1995). Algunos de ellos se utilizaban en los Seminarios de Nobles, las escuelas militares o la Facultad de Filosofía antes de la creación de la segunda enseñanza y aparecerán en las listas oficiales aprobadas a partir de i845.

A partir de 1845 se consolida esta nueva etapa educativa, aunque se mantiene su relación con la Facultad de Filosofía (estudios preparatorios para las facultades mayores). Durante este periodo cada año se publicaron listas de textos a principio de curso, exceptuando el curso i845/1846.

En I857 se aprueba la Ley Moyano, que llevará a cabo una profunda renovación del sistema educativo. En esta etapa comienza una tímida modernización de las ciencias en España, a lo que se intenta contribuir con la creación de la Facultad de Ciencias, aunque el auténtico impulso modernizador se dará a partir de I868 (Peralta, 2009; Etayo, 1992) y alcanzará su clímax a finales de siglo. En I880 se aprueba un nuevo plan de estudios para la Facultad de Ciencias (Plan Lasala) que supuso un impulso para su desarrollo, el cual se vio reflejado en un aumento de las publicaciones (Millán, 199i). En I900 se aprobó el plan de García Alix, que modernizó la Facultad de Ciencias y la equiparó a las de otras naciones europeas (Peralta, 1999). Existen listas de libros de forma continuada hasta I867. Desde esa fecha no aparecen más hasta 1875 , cuando se da permiso a los catedráticos para 
seguir el texto que quieran siempre que lo apruebe la Universidad y no existen nuevas publicaciones hasta los años ochenta.

La Geometría Analítica aparece en los libros de texto utilizados en la segunda enseñanza de la primera mitad del siglo (o en la Facultad de Filosofía, que seguía ligada a ella) y posteriormente, tras su creación en I857, en la Facultad de Ciencias (Vea, 1995; Sánchez, 2015), por lo que son estos niveles educativos a los que hemos restringido nuestro estudio.

Se seleccionaron diez libros siguiendo como principal criterio que la obra estuviera incluida en alguna de las listas oficiales de libros de texto de los planes de estudios de los niveles educativos estudiados. En caso de no haber listas oficiales se tuvo en cuenta la relevancia del autor en la época. A la hora de elegir la edición a consultar se priorizó que estuviera disponible, aunque en algunos casos esta edición quedara fuera del periodo considerado.

Los libros seleccionados son:

- Vallejo, J. M.: Tratado elemental de matemáticas, Madrid, Imprenta de Doña Catalina Piñuela, I817. Fue reeditado por cuarta vez en i84I y aparece en las listas oficiales para los cursos I846/1847 y i847/I848.

- Zorraquín, M.: Geometría analítica-descriptiva, Alcalá, Impresor de la Real Universidad, I8rg. Esta obra fue escrita para la academia militar, pero será recomendada como texto para el estudio de la Geometría Analítica en secundaria y en la Facultad de Ciencias desde el año I847 a I867. Solo hay una edición de este libro.

- Lista, A.: Elementos de matemáticas puras y mistas, tomo III, Madrid, Imprenta de Don León-Amarita, I825. Este libro alcanzó su tercera edición en I838 aunque se ha consultado la segunda.

- Odriozola, J.: Curso completo de matemáticas puras, tomo III, Madrid, Imprenta que fue de García, I829. Este libro fue escrito para la enseñanza militar, pero también se utilizó en la secundaria y aparece en las listas de libros para el curso I846/47.

- Lacroix, S. F.: Curso completo elemental de Matemáticas puras, tomo IV, Madrid, En la Imprenta Nacional, i846. Esta obra aparece como texto en las listas de libros para la Facultad de Filosofía publicadas para los cursos I846/47 y i $847 / 48$.

- Gómez Santa María, A.: Tratado completo de matemáticas, tomo IV, Madrid, Imprenta Nacional, i846. Aparecerá en listas oficiales de textos para la educación secundaria y la Facultad de Ciencias desde i847 a 1867.

- Cortázar, J.: Geometría Analítica, Madrid, Imprenta de Corrales y compañía, I862. Esta obra se encuentra en las listas de libros de texto para la Facultad de Ciencias -donde Cortázar fue catedrático de Geometría Analítica desde 1857 hasta I873- publicadas entre los años I858 y I868.

- Mundi, S.: Lecciones de Geometría Analítica, Barcelona, Establecimiento tipográfico la academia de Evaristo Ullastres, 1883 . Su autor fue catedrático de Geometría Analítica en la Universidad de Barcelona desde i88I. 
CONCEPCIONES DE LA GEOMETRÍA ANALÍTICA EN LOS LIBROS DE TEXTO DE SECUNDARIA Y UNIVERSIDAD EN ESPAÑA DURANTE EL SIGLO XIX ISABEL M. ${ }^{a}$ SÁNCHEZ SIERRA Y M. ${ }^{a}$ TERESA GONZÁLEZ ASTUDILLO

- Sánchez Solís, I.: Geometría Analítica, Madrid, Establecimiento tipográfico de G. Juste, I883. Su autor fue catedrático de Geometría Analítica en la Universidad de Madrid desde 1876 a I89o. No se trata de una obra relevante, es más bien una guía para el profesor, pero suponemos que fue la utilizada en la Facultad de Ciencias de Madrid durante los años en que Sánchez Solís fue catedrático.

- Vegas, M.: Geometría Analítica, Madrid, Establecimiento tipográfico de G. Juste, 1906. Su autor fue catedrático de Geometría Analítica en la Universidad de Madrid desde i89I hasta 1935. Esta obra, editada por primera vez en I894, fue especialmente relevante en el campo de la Geometría de nuestro país, así como su autor.

La crítica fue tanto externa como interna. La externa se basó en el contraste con las listas oficiales de libros publicados para la enseñanza en los niveles educativos correspondientes y los estudios realizados sobre la educación secundaria y la enseñanza de las Matemáticas en este siglo. La crítica interna consistió en un análisis didáctico del contenido (Gómez, 2002; Maz, 2005; Rico et al., 2008; Maz, 2009) de los manuales escolares, fijándonos en tres aspectos: la estructura conceptual, las representaciones externas (símbolos y gráficas) y el análisis fenomenológico (Gómez, 2002), centrándonos en la geometría plana. Tuvimos en cuenta todos estos aspectos, pues las simples definiciones de Geometría Analítica que aportan los autores estudiados no son suficientes para entender las distintas concepciones que aparecen en el s. XIX.

La hermenéutica partió del análisis de las obras teniendo en cuenta unas categorías centradas en el contenido que fueron: las definiciones, las soluciones negativas, el uso del segmento unidad para convertir las ecuaciones heterogéneas en homogéneas, la construcción de las fórmulas, el uso de sistemas de coordenadas, la noción de lugar geométrico, las ecuaciones de la recta y los tipos de problemas resueltos. Todo ello nos ha proporcionado información acerca del código disciplinar de esta asignatura y su evolución.

\section{La Geometría Analítica en los libros de texto en España en el s. XIV}

En las diez obras analizadas hemos encontrado cuatro términos utilizados para nombrar esta parte de las matemáticas relativa a la resolución de proble mas geométricos con ayuda del Álgebra: Aplicación del Álgebra a la Geometría, Geometría Analítica, Análisis (o Geometría Analítica) determinada y Análisis (o Geometría Analítica) indeterminada, aunque estos dos últimos se encuentran incluidos dentro de los anteriores.

Por otra parte, en estos mismos libros hallamos dos formas de resolver problemas geométricos por medio del Álgebra. 
- La primera consiste en resolverlos de forma análoga a los problemas aritméticos, pero operando con segmentos en vez de con cantidades y construyendo geométricamente las soluciones obtenidas algebraicamente.

- La segunda, basándose en el uso de sistemas de coordenadas, de forma similar a la actual.

En relación con el primer método, veremos a lo largo del artículo en qué consiste, a través de las explicaciones que dan los distintos autores, pero se puede resumir de la siguiente forma: se supone el problema resuelto, se asignan letras y números (sus longitudes) a los segmentos implicados en el problema y se establecen relaciones entre ellos mediante propiedades de Geometría elemental que dan una ecuación de ligadura. Se resuelve esta ecuación y se construye geométricamente la expresión algebraica obtenida.

Un ejemplo muy sencillo lo tenemos en el problema de inscribir un cuadrado en un triángulo, resuelto por todos los autores analizados (Sánchez y González, 20I8). Presentamos la solución dada por Lista (I825):

\section{Figura I: Lista, I825, Figura IIo.}
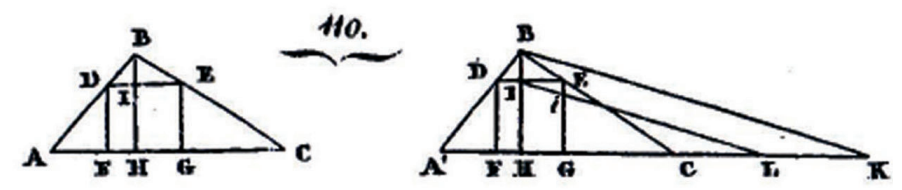

Se supone el problema resuelto siendo el cuadrado DEFG la solución. Si $A C=a$ es la base del triángulo, $B H=b$ la altura del triángulo y $D E=I H=x$ el lado del cuadrado. Al ser los triángulos $A B C, D B E$ semejantes, se obtiene que $\frac{a}{b}=\frac{x}{b-x}$, de donde $x=\frac{a b}{a+b}$. Esta fórmula se construye geométricamente buscando una cuarta proporcional a $a+b, a$ y $b$.

Como hemos dicho, este método de resolución lleva implícitos tres problemas que lo caracterizan: la interpretación de las soluciones negativas, la homogeneidad de las ecuaciones y la construcción de las expresiones algebraicas, que veremos en las obras analizadas.

En la mayoría de las obras estos dos métodos de resolución no se corresponden de forma biunívoca con los términos señalados más arriba, sino que nos encontramos con cuatro combinaciones de estos:

- Se utiliza únicamente el término Aplicación del Álgebra a la Geometría y este engloba a las dos formas citadas de resolver los problemas. 
CONCEPCIONES DE LA GEOMETRÍA ANALÍTICA EN LOS LIBROS DE TEXTO DE SECUNDARIA Y UNIVERSIDAD EN ESPAÑA DURANTE EL SIGLO XIX

ISABEL M. ${ }^{a}$ SÁNCHEZ SIERRA Y M. ${ }^{a}$ TERESA GONZÁLEZ ASTUDILLO

- Se utilizan los términos Aplicación del Álgebra a la Geometría y Geometría Analítica para denominar indistintamente a esas dos formas, pero los autores distinguen entre Análisis (o Geometría Analítica o problemas) Determinada e Indeterminada. Bajo la primera acepción se incluye el primer método que hemos citado y bajo la segunda lo que hoy entendemos por Geometría Analítica.

- Se utiliza el término Aplicación del Álgebra a la Geometría para referirse a la primera acepción y Geometría Analitica para la segunda.

- Se utiliza únicamente el término Geometría Analítica, pero en esas obras solo aparece la geometría que utiliza los sistemas de coordenadas.

Analizaremos a continuación cada uno de estos casos y los autores que muestran dichas concepciones a través de sus libros.

\section{I. Aplicación del Álgebra a la Geometría}

Encontramos esta denominación en las obras de Vallejo (I8I7), Lista (I825) y Lacroix (I846). Ninguno utiliza el término Geometría Analítica, con la diferencia de que Vallejo (I8I7) y Lacroix (I846) incluyen los dos tipos de geometría de los que hemos hablado y Lista (I825), sin embargo, no trabaja con sistemas de coordenadas.

Vallejo (I8I7) define la Aplicación del Álgebra a la Geometría de la siguiente manera:

95. El Algebra es mas general que la Geometría, porque con la letra a, por egemplo, podemos espresar no solo una cantidad cualquiera discreta, sino una cantidad continua como una linea, una superficie \&c. La Geometría es mas clara que el Algebra, porque presenta á los sentidos los objetos de las lineas que forman el asunto de nuestras investigaciones. Cuando para generalizar ó facilitar alguna verdad geométrica se hace uso del Algebra ó de la análisis algebráica, se dice que se aplica el Algebra á la Geometría; y cuando para hacer sensible alguna verdad algebráica ó analítica se hace uso de la Geometría, se dice que se aplica la Geometría al Algebra ó á la análisis. Pero en general se comprende bajo el nombre de Aplicacion del Algebra á la Geometría, al uso que se hace de estas dos ciencias, y sea para resolver alguna cuestion perteneciente á una de ellas, ya para resolver cualquier otra, sea de la especie que sea (Vallejo, I817: 68).

Esta definición tan genérica no muestra la manera en que Vallejo desarrolla su geometría, pero analizando el contenido de la obra vemos las dos acepciones que hemos señalado.

En el primer capítulo observamos la primera de ellas, pues utiliza el Álgebra para resolver problemas geométricos de manera similar a como se utiliza para resolver los aritméticos, pero operando con segmentos en vez de con cantidades. Vallejo (I8I7) explica cómo llevar a cabo la construcción con regla y compás de 
CONCEPCIONES DE LA GEOMETRÍA ANALÍTICA EN LOS LIBROS DE TEXTO DE SECUNDARIA Y UNIVERSIDAD EN ESPAÑA DURANTE EL SIGLO XIX ISABEL M. ${ }^{a}$ SÁNCHEZ SIERRA Y M. ${ }^{a}$ TERESA GONZÁLEZ ASTUDILLO

las diferentes operaciones algebraicas, la necesidad de que las ecuaciones sean homogéneas y la cuestión de la validez de las soluciones negativas.

Sin embargo, tras resolver varios problemas llega a la conclusión de que el método explicado no es suficiente para resolver todos los tipos de problemas geométricos que se puedan plantear, por lo que se hace necesario introducir otro más general:

I32. Más para buscar un método que asegure la posibilidad de poner todos los problemas en ecuacion y de un modo uniforme, consideraremos que una cuestion de Geometría se puede reducir siempre en última análisis á encontrar uno ó muchos puntos dispuestos en virtud de ciertas condiciones dadas; de donde se sigue que para cumplir con el objeto propuesto, es necesario indagar como se puede espresar analíticamente la posición de los puntos del espacio (Vallejo, i8ı7: 82).

Así, en el siguiente epígrafe trabaja con sistemas de coordenadas (rectangulares, oblicuas y polares), obteniendo la ecuación de una recta y resolviendo problemas relativos a los puntos y las rectas de un plano (incidencia, perpendicularidad y paralelismo, ángulos y distancias), de forma muy similar a la actual.

Vallejo (I8I7) no utiliza el término lugar geométrico, pero sí explica la relación que puede establecerse entre una ecuación y una línea indicando que de igual manera que se puede expresar la posición de un punto analíticamente podemos hacerlo para todos los puntos de una línea:

Generalizando este resultado se ve que si todos los puntos de una linea cualquiera, recta ó curva, son tales que existe la misma relacion entre las ordenadas y las abscisas de cada uno de ellos, la ecuacion entre $x$ y $z$ que esprese esta relacion, debe caracterizar á esta linea. [...]

I50. Una ecuacion que espresa de este modo la relacion que tienen entre sí las abscisas y las ordenadas de cada punto de una linea, se llama la ecuacion de esta linea; y esta es continua ó discontinua, según convenga ó no convenga la misma ecuacion á todos los puntos de que está compuesta (Vallejo, ı8ı7: 88).

Por su parte, Lacroix (I846) dedica el tercer capítulo de su obra a la Aplicación del Álgebra a la Geometría. En él da tres aplicaciones del álgebra a la geometría, la primera de ellas ya utilizada en capítulos anteriores de su obra:

El objeto de la aplicación del álgebra á la geometría es que haciendo uso de las operaciones algebraicas, se pueden combinar muchos teoremas de geometría, y deducir de estas combinaciones consecuencias importantes. Este es el camino que hemos seguido en los dos capítulos anteriores, y por él hemos llegado á sacar las principales fórmulas de las trigonometrías rectilínea y esférica. Un teorema, que establece una relacion entre muchas líneas de una magnitud definida, puede expresarse siempre por una ecuacion; y todas las transformaciones que se hacen en tal ecuacion, cuando se las traduce al lenguaje comun, dan enunciados que son consecuencias del teorema de que se ha partido (Lacroix, I846: 99). 
Es decir, mediante el paso al Álgebra se pueden simplificar los teoremas de la Geometría, y además deducir de las transformaciones realizadas en las ecuaciones nuevos teoremas. En este tercer capítulo introduce otro tipo de aplicación del álgebra a la geometría, y así lo aclara a continuación:

Este ramo de las matemáticas, considerado en general, no se limita á la investigación de las propiedades de la extensión por medio de las operaciones algebráicas, sino lo que es mas, cómo se puede representar por estas propiedades lo que significa una expresión algebráica cualquiera, reducir las construcciones de las figuras á las operaciones del cálculo, y pasar de estas á las primeras: todo esto lo manifestarán las diversas cuestiones tratadas en este capítulo (Lacroix, I846: 99).

Se dará la interpretación geométrica de una ecuación algebraica para que a la hora de resolver un problema geométrico se pase este a lenguaje algebraico y una vez hechas las operaciones pasar de nuevo a la construcción geométrica de la solución algebraica obtenida. Vemos por tanto la primera de las concepciones citadas.

Tras desarrollar este método de resolución de problemas inicia una nueva forma de trabajar utilizando las ecuaciones de las curvas, comenzando por explicar este nuevo enfoque:

8r. No solo sirve el Algebra para hallar la magnitud de las líneas y de las partes de la extension, comparadas las unas á las otras, sino que da tambien el medio de determinar las figuras que forman estas líneas, y en general las formas del espacio. Notando Descartes, el primero, que estas figuras y estas formas establecen relacion de magnitud entre las líneas, llegó á aplicar el algebra á la teoría de las líneas en general; y por este descubrimiento cambiaron de aspecto las Matemáticas (Lacroix, I846: I32).

Así, las curvas se pueden expresar mediante ecuaciones algebraicas, concepto fundamental de la Geometría Analítica. Es más, Lacroix (1846) habla explícitamente de lugar geométrico y explica qué es:

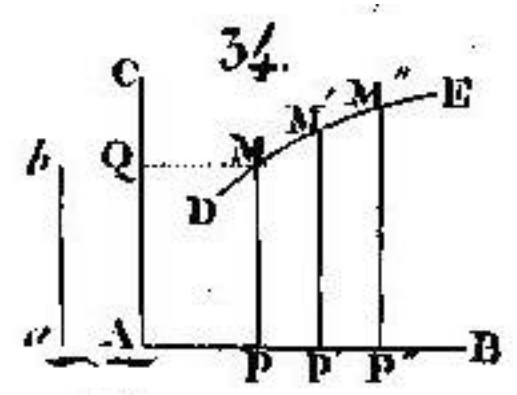

86. La ecuacion que expresa las relaciones entre las AP y las $\mathrm{PM}^{1}$ para una línea dada, se llama la ecuacion de esta linea, y esta se llama el lugar geométrico de la ecuacion que la perten

AP y PM son la abscisa y la ordenada, respectivamente, de un punto M de la línea. 
CONCEPCIONES DE LA GEOMETRÍA ANALÍTICA EN LOS LIBROS DE TEXTO DE SECUNDARIA Y UNIVERSIDAD EN ESPAÑA DURANTE EL SIGLO XIX ISABEL M. ${ }^{a}$ SÁNCHEZ SIERRA Y M. ${ }^{a}$ TERESA GONZÁLEZ ASTUDILLO

Es claro que toda cuestion geométrica indeterminada que contenga dos incógnitas, conduce á un lugar geométrico (Lacroix, 1846: 138-139).

Hay que señalar que Lacroix es el único autor de los analizados que resuelve el mismo problema utilizando ambos métodos (Sánchez y González, 20ı8).

En cuanto a Alberto Lista (I825), dedica un capítulo a la Aplicación del álgebra a la Geometría. En la definición establece las partes de que consta la solución analítica de un problema geométrico y las ventajas del método que va a utilizar:

I. Llámase aplicación del álgebra á la geometría la solución de los problemas geométricos por medio de ecuaciones algebraicas.

2. La solución analítica de un problema geométrico consta de cuatro partes: $\mathrm{I}^{\mathrm{a}}$, representar por letras los datos y las incógnitas: $2^{a}$, poner el problema en ecuación, ya por las propiedades de la figura geométrica, ya por las condiciones del problema: $3^{\text {a }}$, despejar las incógnitas: $4^{\text {a }}$ construir las fórmulas, es decir, hacer las operaciones geométricas que indica el valor de la incógnita (Lista, I825: I2I).

En este capítulo se define también dimensión de una ecuación, ecuaciones homogéneas y heterogéneas, explica cómo construir geométricamente las expresiones algebraicas (Sánchez y González, 2017) y resuelve varios problemas, pero no hace referencia en ningún momento, como hemos señalado, a los sistemas de coordenadas ni al concepto de lugar geométrico.

\subsection{Aplicación del Álgebra a la Geometría o Geometría Analítica}

Encontramos el uso indistinto de estos dos términos en las obras de Zorraquín (I819), Odriozola (I829) y Gómez Santa María (1846). Además, en todas estas obras aparecen las dos concepciones de Geometría Analítica que hemos visto, pero hay que señalar que todos hacen una distinción entre ellas, utilizando los términos «determinada» e «indeterminada».

La obra de Zorraquín (I8I9) está divida en dos secciones que delimitan claramente los dos tipos de Geometría Analítica que estamos tratando. La primera se denomina Análisis Determinada e incluye a la aplicación del álgebra a la Geometría sin uso de sistemas de coordenadas, y la segunda Análisis Indeterminada, que es aquella que sí las utiliza.

Comienza planteando la posibilidad de resolver los problemas geométricos con ayuda del Álgebra de forma similar a como se hace con los aritméticos y señala:

Recordaremos á este fin que las magnitudes lineales en tanto tienen existencia en cuanto se las refiere á una unidad de medida; esta no es una consideración arbitraria, pues sin ella nada significaría el producto de dos líneas, el cuadrado de una \& (Zorraquín, I819: 2). 
CONCEPCIONES DE LA GEOMETRÍA ANALÍTICA EN LOS LIBROS DE TEXTO DE SECUNDARIA Y UNIVERSIDAD EN ESPAÑA DURANTE EL SIGLO XIX

ISABEL M. ${ }^{a}$ SÁNCHEZ SIERRA Y M. ${ }^{a}$ TERESA GONZÁLEZ ASTUDILLO

Y teniendo en cuenta esto, es fácil convertir un problema geométrico en otro algebraico:

Si pues siempre que entren en los cálculos, substituimos por ellas los números que expresan sus relaciones con la unidad las cuestiones geométricas podrán considerarse como numéricas, y resolverse por el Álgebra empleando sus caracteres generales para representar dichas magnitudes. [...] Delo dicho resulta que si después de averiguar las relaciones que existen entre las magnitudes dadas y las que se buscan las ciframos en una ecuación, resolviéndola llegaremos al resultado sin el trabajo que de otro modo sería indispensable (Zorraquín, I8ıя: 3).

E indica que «Esto es lo que se entiende por Aplicación del Algebra á la Geometría» (p. 3), señalando que juntas se ayudan mutuamente, el Álgebra facilitando y dando generalidad a los problemas de Geometría y la Geometría interpretando las soluciones algebraicas, ya que «en toda expresión analítica debemos ver una construcción geométrica pues que sus caracteres representarán cantidades geométricas» (p. 3).

Tras esto concluye: «Dos son pues los objetos de la Geometría analítica; resolver las cuestiones geométricas por el análisis é interpretar geométricamente ó construir las fórmulas analíticas» (p. 4).

En la sección dedicada a la Análisis Determinada se trata la homogeneidad de las ecuaciones al aplicarlas en geometría, cómo se construyen las fórmulas de primer y segundo grado, se estudia la interpretación de las soluciones negativas de un problema geométrico y se resuelve varios problemas (Sánchez y González, 2016).

La sección dedicada a la Análisis Indeterminada comienza con una introducción en la que da la noción de otro método de resolución de problemas, distinto y más general que el explicado en la sección I.

34. Las cuestiones propuestas hasta ahora y cuantas se propongan relativas á la Geometría pueden resolverse de un modo mas general y favorable á las investigaciones. En efecto, entendiendo por espacio la extensión en que existen los cuerpos, indefinida, sin límites, y cuyas partes son todas semejantes entre sí, supongamos determinados en él algunos objetos fijos á que referiremos los demás. Si por los datos de una cuestión ó de otro cualquier modo es conocida la posición respecto de ellos de cada uno de los puntos del objeto que se busca, y se los coloca en el orden que corresponde, es claro que llegaremos á trazarle y describirle completamente, y que por esta descripción se obtendrá su figura y magnitud. Vemos pues que los Problemas de Geometría pueden por último reducirse á situar un punto en la posición que eligen ciertas condiciones (Zorraquín, I8ı9: 49).

En esta sección desarrolla la idea de cómo situar un punto en un plano y cómo representar una línea usando el álgebra; trata acerca de los sistemas de referencia, y de los sistemas de coordenadas oblicuas, rectangulares y polares. Tras estas consideraciones generales analiza las ecuaciones de primer grado entre dos va- 
riables. Estudia la ecuación de una recta en un plano, dando diferentes tipos de ecuaciones y halla el punto de corte de dos rectas, la distancia entre dos puntos y el ángulo que forman dos rectas, entre otras cuestiones, de forma similar a como se hace en la actualidad.

En cuanto a la obra de Odriozola (I829), la Geometría Analítica aparece en el tomo tres, que se subtitula Álgebra sublime y Geometría Analítica. Odriozola lo divide en dos tratados que se corresponden con el subtítulo del tomo, en concreto uno de ellos se titula Geometría Analítica ó Aplicación del Algebra a la Geometría. Sin embargo, hace una distinción entre Ecuaciones determinadas $y$ Ecuaciones indeterminadas, para referirse con las primeras a aquella manera de hacer Geometría Analítica que no requiere de sistemas de coordenadas y las segundas para las ecuaciones de las curvas. Vemos, por tanto, dos partes claramente diferenciadas por metodología y contenido, y dos términos entre los que no se hace distinción.

Empieza el tratado con una lección preliminar en la que explica, entre otras cosas, las aplicaciones del Álgebra a la Geometría y viceversa:

2. Desde ahora vamos á tratar de las reciprocas aplicaciones de una ciencia á la otra; y por el resultado se formará el juicio de que mas necesita del Algebra la Geometría, que de ésta la primera.

[...] y estan resumidas en dos proposiciones generales: I. $^{a}$ cifrar en lenguaje de cálculo las cuestiones y resolverlas: $2 .{ }^{a}$ construir espresiones del cálculo, ó traducirlas á lenguaje de figuras geométricas (Odriozola, I829: 180).

Termina recordando la regla general para pasar del lenguaje geométrico al algebraico que consiste en suponer el problema resuelto, $\mathrm{y}$ «nombrando con cifras propias las cantidades, escribir la oracion para despues resolver segun los principios del cálculo» (p. I8I).

En cuanto a las ecuaciones determinadas se analiza la construcción de estas ecuaciones, es decir, de las expresiones algebraicas. Como otros autores de la época, Odriozola insiste en la homogeneidad de las ecuaciones, así como en la interpretación de las soluciones negativas de una ecuación en el contexto geométrico. Se resuelven varios problemas utilizando este método.

Para las ecuaciones indeterminadas, que no son más que las ecuaciones de la recta, utiliza lo que él llama sistemas de coordenadas rectas -que pueden ser oblicuos y rectangulares- y las coordenadas polares. Tras esto construye la ecuación de primer grado en dos variables e interpreta el significado de las constantes que en ella aparecen, llegando a la conclusión de que representa una recta, dando diferentes expresiones de esta. Además, presenta diferentes problemas de ángulos, posiciones relativas y distancias, de manera similar a la actual.

El tomo cuarto de la obra de Santa María (1846) se subtitula Geometría Analítica ó Aplicación del Análisis a la Geometría. El autor utiliza ambos términos indistintamente y también aparecen las dos concepciones, pero, de forma análoga 
CONCEPCIONES DE LA GEOMETRÍA ANALÍTICA EN LOS LIBROS DE TEXTO DE SECUNDARIA Y UNIVERSIDAD EN ESPAÑA DURANTE EL SIGLO XIX

ISABEL M. ${ }^{a}$ SÁNCHEZ SIERRA Y M. ${ }^{a}$ TERESA GONZÁLEZ ASTUDILLO

a Zorraquín (I8I9) y Odriozola (I829), las distingue utilizando los términos problemas determinados e indeterminados.

El libro comienza explicando cómo se suman, restan, multiplican y dividen las líneas gráficamente y con ello cómo se puede aplicar el Álgebra a la Geometría de manera similar a como se aplica a la Aritmética:

Este recuerdo de los procedimientos gráficos para obtener sumas, restas, productos y cocientes entre rectas (procedimientos que no excluyen el poder espresar por números, y operar con ellos, obteniendo por resultado un nuevo número que seria el valor de la recta obtenida por los medios directos), conduce á entrever que así como el álgebra se aplica á cuestiones de números, del mismo modo se podrá emplear en las de líneas.

[...] se reconoce inmediatamente que reemplazando de la misma manera por signos cualesquiera, como lo son las letras, las diversas líneas que han de producir otra por medio de una combinación oportuna, en calculando sobre dichos signos con buen raciocinio, es decir, según las reglas del álgebra, se llegará á obtener un resultado final ó fórmula que dará la línea que se busca tan luego como se construya ó forme la figura geométrica que está representando la combinación de las líneas dadas.

Por consiguiente la diferencia entre la aplicacion del álgebra á las cuestiones numéricas y la aplicación á las geométricas, solo existe en la interpretación que se $\mathrm{da}$ á los resultados [...] en el segundo caso los resultados son y han de mirarse como líneas, que quedarán trazadas en el mismo momento que se levante y arme, por decirlo así, con la regla y el compás la figura que está escrita en la combinación de líneas de dichos resultados ó fórmulas (Gómez de Santa María, I846: 2).

También se define dimensión de una expresión algebraica, ecuación homogénea y unidad. Tras esto se explica la construcción geométrica de distintas expresiones algebraicas y se indica cómo construir e interpretar geométricamente las soluciones negativas de un problema. Seguidamente se resuelven varios problemas «determinados» de Geometría en los que muestra la aplicación de las construcciones y conceptos explicados en las secciones anteriores.

En el capítulo IV, titulado Problemas indeterminados. Ecuaciones del punto y de las rectas en el plano, se tratan los problemas relativos a las ecuaciones de una recta. Se comienza con la posición de un punto en un plano, se definen los ejes de coordenadas (oblicuos y rectangulares) y las coordenadas de un punto. Tras esto se llega a la conclusión de que una ecuación en dos variables representa a una línea y se estudian diferentes casos particulares como son las ecuaciones de los ejes de coordenadas y de rectas paralelas a ellos, generalizando hasta dar la ecuación explícita de una recta. Se dan diferentes expresiones de la misma, entre ellas la ecuación general de una recta, a la que se llega tras definir el concepto de lugar geométrico.

20. Por lugar geométrico de una ecuacion se entiende, la línea cuyas coordenadas tengan la relacion que esprese la ecuación dada. 
Toda ecuacion de primer grado entre dos variables, tiene por lugar geométrico una recta (Gómez de Santa María, I846: 74).

Además, se estudia el ángulo que forman dos rectas, se obtienen las condiciones de paralelismo y perpendicularidad y la distancia entre dos puntos.

\subsection{Aplicación del Álgebra a la Geometría versus Geometría Analítica}

Solamente encontramos esta distinción en el texto de Cortázar (I862). A pesar del título del libro, Cortázar hace una distinción muy clara entre lo que es la aplicación del Álgebra a la Geometría elemental y la Geometría Analítica propiamente dicha, definiendo de forma independiente en qué consiste cada una de ellas.

I. Se llama aplicación del Algebra á la Geometría elemental la ciencia que trata de la resolución de las cuestiones de la geometría elemental por medio del cálculo algébrico ordinario.

Para que las cuestiones de Geometría puedan resolverse por medio del cálculo algébrico, es menester representar algébricamente las magnitudes de las líneas y superficies limitadas y la de los espacios que ocupan los cuerpos (Cortázar, I862: I).

Mientras que define Geometría Analítica como:

Podemos ya definir la Geometría analítica plana, diciendo que es la ciencia que se ocupa del estudio dé las líneas planas por métodos generales, representándolas antes por medio de ecuaciones (Cortázar, 1862: 47).

Tenemos así que la obra está dividida en tres partes, la primera dedicada a dicha aplicación, que subtitula Introducción al estudio de la Geometría Analítica, y las dos siguientes, tituladas Geometría Analítica Plana y Geometría Analítica del Espacio respectivamente.

En la primera, que es muy breve en relación con las otras, se explica cómo se pueden representar algebraicamente las cantidades geométricas, lo que lleva al estudio de las ecuaciones homogéneas y del segmento unidad, problema que se resuelve mediante la demostración de un teorema (p. 5), lo que contrasta con obras anteriores donde mostraban el uso del segmento unidad mediante ejemplos, y resuelve problemas «de Geometría Elemental».

En cuanto a la parte dedicada a la Geometría Analítica plana, se presentan los sistemas de coordenadas rectangulares y oblicuas, las ecuaciones de la recta y se resuelven problemas de distancias. También se define el concepto de lugar geométrico de una ecuación: 
20. Dada una ecuación con dos incógnitas ó variables $x$ é $y$, para hallar sus diferentes soluciones, se despeja una de las incógnitas, $y$ por ejemplo, se dan á la otra, $x$, valores arbitrarios, y se hallan los valores correspondientes de la $y$.

Supongamos ahora que se construyan los puntos, cuyas coordenadas sean las diferentes soluciones halladas: como estos puntos pueden construirse tan próximos entre si como se quiera, formarían una línea. Luego á toda ecuacion con dos variables corresponde (prescindiendo de los casos de escepcion, que mas adelante examinaremos) una línea plana que se llama el lugar geométrico de la ecuación (Cortázar, I862: 45).

Vemos en esta obra, por tanto, una pequeña evolución dentro del estudio de la Geometría Analítica, aunque el gran salto lo encontramos en las obras publicadas a partir de i88o.

\subsection{Geometría Analítica}

Las obras de Mundi (I883), Sánchez Solís (I883) y Vegas (I906) son radicalmente distintas a las de los años anteriores. En ellas la Geometría Analítica se estudia englobada dentro de la Geometría Proyectiva.

En las tres obras solo aparece el término Geometría Analítica, pero en la de Sánchez Solís (I883) se establece la distinción entre Geometría Analítica determinada e indeterminada, y es en ella en la única que aparecen algunos vestigios de la primera de las concepciones de las que hemos hablado, bajo la primera denominación. La Geometría Analítica Indeterminada se engloba dentro de la Geometría Proyectiva, como ya hemos dicho.

Solís (i883) comienza su obra con la definición de Geometría Analítica y las partes de que consta:

La Geometría Analítica es la parte de las Matemáticas que enlaza el Algebra con la Geometría para utilizar en la resolución de las cuestiones la generalidad de la primera y la claridad de la segunda. Puede dividirse en determinada é indeterminada, y ésta en plana y del espacio, ó sea de dos y de tres dimensiones (Sánchez Solís, 1883: 3).

Y así es como está dividida la obra, haciendo distinción entre las dos geometrías, aunque a la primera solamente le dedica cinco páginas en las que explica de forma sucinta los pasos para resolver un problema mediante este método y cómo se construyen algunas expresiones algebraicas.

En la parte dedicada a la Analítica Indeterminada presenta diferentes sistemas de coordenadas, no solamente las coordenadas cartesianas y polares, sino también las tangenciales binarias, las coordenadas trilineales y las tangenciales ternarias. Se resuelven problemas relativos a distancias, ángulos e incidencia y lo más significativo es que introduce conceptos propios de la Geometría Proyectiva. 
CONCEPCIONES DE LA GEOMETRÍA ANALÍTICA EN LOS LIBROS DE TEXTO DE SECUNDARIA Y UNIVERSIDAD EN ESPAÑA DURANTE EL SIGLO XIX ISABEL M. ${ }^{a}$ SÁNCHEZ SIERRA Y M. ${ }^{a}$ TERESA GONZÁLEZ ASTUDILLO

Sin embargo, ni en la obra de Mundi (I883) ni en la de Vegas (1906) existen restos de lo que algunos autores han llamado Análisis Determinada.

Para Mundi (I883) «la Geometría analítica estudia las propiedades de las figuras geométricas por medio del cálculo algébrico» (p. 7), señalando que "Toda línea es un lugar geométrico de puntos que gozan de una misma propiedad expresada por la definición» (p. I5).

Introduce los sistemas de coordenadas cartesianas, polares, tangenciales y trilineales, estudiando en cada uno de ellos las ecuaciones de la recta y problemas de incidencia, paralelismo y perpendicularidad, ángulos y distancias. Es de destacar la introducción de conceptos propios de la Geometría Proyectiva tales como recta y punto del infinito, sistemas armónicos o involución.

La obra de Vegas (I906) es conceptualmente bastante compleja pues, aunque es un libro sobre Geometría Analítica, se plantea desde el punto de vista de la Geometría Proyectiva, por lo que se utilizan continuamente los conceptos propios de esta última para definir los de la primera. Así, por ejemplo, no solo se utiliza la ley de correlación para relacionar las coordenadas cartesianas con las tangenciales, sino que estos sistemas se estudian como casos particulares de otros más generales, definidos todos ellos utilizando los conceptos de Geometría Proyectiva.

Se define la Geometría Analítica al comienzo de la obra:

4. El estudio de las propiedades de las figuras geométricas constituye el objeto de la Geometría, y cuando este estudio se verifica, utilizando como medio el Análisis matemático, recibe el nombre de Analítica. La Geometría analítica se propone, pues, estudiar las figuras geométricas utilizando los recursos del Álgebra, ó sea, dándolas representación algébrica, con lo cual se consigue dar á las cuestiones geométricas la generalidad del Análisis; pero a su vez, permite pasar del Álgebra á la Geometría, dando á las cuestiones analíticas el sabor geométrico que tanto contribuye a la claridad (Vegas, 1906: 2).

Y su estudio se ciñe en esta obra al uso de sistemas de coordenadas (coordenadas binarias y ternarias del punto y la recta, coordenadas polares, polares tangenciales y tripolares), el estudio de la ecuación de la recta o el punto (en coordenadas tangenciales) y la resolución de problemas de incidencia, distancias y ángulos.

Como vemos estas obras representan un salto cualitativo muy importante en el estudio de la Geometría Analítica en España, respecto a las obras utilizadas hasta el último cuarto de siglo, y aunque el concepto de Geometría Analítica es básicamente el mismo en todas ellas -el uso conjunto de la Geometría y el Álgebra- el enfoque es radicalmente distinto. 
CONCEPCIONES DE LA GEOMETRÍA ANALÍTICA EN LOS LIBROS DE TEXTO DE SECUNDARIA Y UNIVERSIDAD EN ESPAÑA DURANTE EL SIGLO XIX

ISABEL M. ${ }^{a}$ SÁNCHEZ SIERRA Y M. ${ }^{a}$ TERESA GONZÁLEZ ASTUDILLO

\section{Conclusiones}

La Geometría Analítica que surge en el siglo xvir distaba bastante de lo que entendemos actualmente. Su evolución a lo largo de los siglos convirtió la primera en la segunda, siendo el siglo XIV un hito importante en el camino de esta evolución, hito que hemos estudiado en nuestro país mediante el análisis de los libros de texto utilizados en el periodo.

A través de este análisis hemos encontrado dos formas distintas de hacer Geometría Analítica: uno más próximo a la Geometría de Descartes que a la actual, que resuelve los problemas geométricos por medio del Álgebra de forma análoga a como se resuelven los problemas aritméticos, pero operando con segmentos en vez de con cantidades y construyendo la solución geométricamente; y otro muy similar a lo que hoy entendemos por Geometría Analítica basado en el uso de sistemas de coordenadas y el concepto de lugar geométrico. Y cuatro términos para designarlas, englobados en dos -Aplicación del Álgebra a la Geometría y Geometría Analitica-, que no se corresponden biunívocamente en la mayoría de las obras con esas dos formas de entender la Geometría Analítica.

Podemos observar también la evolución seguida por la Geometría Analítica durante este siglo, tanto en denominación como en contenido, siendo el punto de inflexión la obra de Cortázar, que distingue claramente en Aplicación del Álgebra a la Geometría -para referirse al primer tipo de Geometría que hemos señalado- y Geometría Analítica para la segunda.

Esta evolución se observa en el tratamiento que se hace de este tema entre los libros de secundaria de la Facultad de Ciencias. En estos últimos se va evolucionando hacia un tratamiento más algebraico y menos geométrico y, hacia el final del periodo analizado, el tratamiento de la Geometría Analítica como parte de la Geometría Proyectiva.

En las obras anteriores encontramos los dos tipos de Geometría que hemos citado (excepto en la obra de Lista), pero utilizando solamente el término Aplicación del Álgebra a la Geometría -caso de Lista, Vallejo y Lacroix- o utilizando este y el de Geometría Analítica para referirse a ambas indistintamente, como hemos visto en las obras de Zorraquín, Santa María y Odriozola. Estos autores distinguen entre Determinada para referirse a la primera e Indeterminada, que es la que hace uso de los sistemas de coordenadas.

Las obras posteriores a Cortázar, ya en el último cuarto de siglo, solo utilizan el término Geometría Analítica y la Analítica Determinada aparece como algo residual únicamente en la obra de Sánchez Solís. La obra de Mundi y especialmente la de Vegas, influenciadas por la ciencia europea que en aquellos momentos está llegando a España, dan un nuevo enfoque a la Geometría Analítica, tratándola como una parte de la Proyectiva y sin conservar vestigio alguno de aquella Geometría Analítica que conectaba con la Geometría de Descartes. 
CONCEPCIONES DE LA GEOMETRÍA ANALÍTICA EN LOS LIBROS DE TEXTO DE SECUNDARIA Y UNIVERSIDAD EN ESPAÑA DURANTE EL SIGLO XIX ISABEL M. ${ }^{a}$ SÁNCHEZ SIERRA Y M. ${ }^{a}$ TERESA GONZÁLEZ ASTUDILLO

A lo largo del siglo xix, por lo tanto, la concepción de Geometría Analítica evolucionó desde una visión puramente geométrica en la que se mantenían prácticas anteriores y que para resolver problemas se utilizan la geometría y el álgebra conjuntamente, a una visión más estrictamente algebraica en la que se fue poco a poco desprendiendo de aspectos e interpretaciones geométricas para reducir la resolución de problemas a una cuestión puramente algebraica. Un concepto esencial en esta evolución fue la presencia de la noción de lugar geométrico primero de forma esporádica en algunos libros de los periodos iniciales que fue asentándose hasta hacerse imprescindible en los últimos libros del siglo estudiado.

\section{Bibliografía}

Chica, A.: La matemática en sus personajes. Descartes. Geometría y método, Madrid, Nivola, $200 I$.

Choppin, A.: «Los manuales escolares de ayer a hoy: el ejemplo de Francia», Historia de la Educación, I9 (2000), pp. 13-37.

Collados, E.: «El concepto de dibujo y su práctica en los libros de texto de educación primaria publicados en España en el periodo comprendido entre 1915-1900», Historia de la Educación, 27 (2008), pp. 323-346.

Delgado, B.: «Los libros de texto como fuente para la historia de la Educación», Historia de la Educación, 2 (1983), pp. 353-358.

Descartes, R.: La Geometría, traducido por Pedro Rossell, Buenos Aires, Espasa-Calpe, I947 (1637).

Etayo, J. J.: «El reinado de la Geometría Proyectiva», en Historia de la Matemática, 1992, pp. IIS-I38.

Fermat, P. : Oeuvres de Fermat, eds. Henry, C. y Tannery, P., París, Gauthier-Villars, I89II9I2.

Gaita, R. C.: El paso de la Geometría sintética a la Geometría analítica, Tesis doctoral, Universidad de Valladolid, 2014.

Gómez, P. (2002): «Análisis didáctico y diseño curricular en matemáticas», Revista EMA, 7 (3) (2002), pp. 25I-292.

González, P. M.: «Raíces históricas y trascendencia de la Geometría analítica», SIGMA, 30 (2007), pp. 205-236.

Goodson, I. F.: Historia del currículum. La construcción social de las disciplinas escolares, Barcelona, Pomares-Corredor, 1995.

MAz, A.: Los números negativos en España en los siglos XVIII y XIX, Tesis doctoral, Universidad de Granada, 2005.

Maz, A.: «Investigación histórica de conceptos en los libros de matemáticas», en González, M. J.; GonzÁlez, M. T. y Murillo, J. (eds.): Investigación en Educación Matemática XIII, Santander, SEIEM, 2009, pp. 5-20.

Millán, A.: «Los estudios de Geometría Superior en España en el siglo XIX», Llull, Revista de la Sociedad Española de Historia de las Ciencias, I4 (I991), pp. II7-I86.

Peralta, J.: La matemática española y la crisis de finales del siglo xix, Madrid, Nivola, 1999.

Peralta, J.: «La matemática española del siglo XIX», en Fundación Orotava de Historia de la Ciencia (ed.): La ciencia antes de la gran guerra. Actas año XviI. Encuentros educativos, Canarias, España: Imprenta Reyes, S.L., 2009, pp. 2II- 236. 
Puelles, M.: «Los manuales escolares: un nuevo campo de conocimiento», Historia de la Educación, I9 (2000), pp. 5-II.

Rico, L.; Marín, A.; Lupiáñez, J. L. y Gómez, P.: «Planificación de las matemáticas escolares en secundaria. El caso de los números naturales», SUMA, 58 (2008), pp. 7-23.

Ruiz Berrio, J.: «El método histórico en la investigación histórica de la educación», Revista Española de Pedagogía, I34 (1997), pp. 449-475.

SÁnchez, I. M.: La Geometría Analítica en los libros de texto para secundaria y universidad en España en el siglo XIX, Tesis doctoral, Universidad de Salamanca, 20I5.

SÁnchez, I. M y González, M. T.: «La Geometría Analítico-Descriptiva de Mariano Zorraquín», HISTEMAT, v. 2, n. 3 (2016), pp. 200-228.

SÁnchez, I. M y González, M. T.: «La Geometría Analítica en España durante el siglo xix: estudio de las soluciones negativas de una ecuación», Enseñanza de las Ciencias, 35 (3) (2017), pp. 89-106.

Sánchez, I. M y González, M. T.: «Inscribir un cuadrado en un triángulo. Métodos de resolución en libros de Geometría Analítica del siglo XIX», SUMA, 89 (2018), pp. 9-17.

Schubring, G.: "On the Methodology of Analysing Historical Textbooks: Lacroix as Textbook Author», For the Learning of Mathematics, 7 (3) (1987), pp. 4I-5I.

VEA, F.: Las matemáticas en la enseñanza secundaria en España en el siglo XIX, Zaragoza, Seminario de Historia de la Ciencia y de la Técnica de Aragón. Facultad de Ciencias (Matemáticas), 1995 .

ViÑaO, A.: «La Historia de las disciplinas escolares», Historia de la Educación, 25 (2006), pp. 243-269. 
